Original article

\title{
Seroprevalence of hydatid cyst and related risk factors in humans referred to urban health centers of Zahedan city, southeast Iran
}

\author{
Samaneh Abdolahi Khabisi ${ }^{\text {a,b, }}$, Malihe Marghzari ${ }^{\text {a }}$, Seideh Zeinab Almasi ${ }^{\text {c, }}$ \\ Alireza Salimi Khorashad ${ }^{\text {a }}$, Soudabeh Etemadi ${ }^{\text {a,b }}$ \\ ${ }^{a}$ Dept. of Parasitology and Mycology, Faculty of Medicine, Zahedan University of Medical Sciences, Zahedan, Iran \\ ${ }^{\mathrm{b}}$ Infectious Diseases and Tropical Medicine Research Center, Zahedan University of Medical Sciences, Zahedan, Iran \\ ${ }^{\mathrm{c}}$ Department of Epidemiology and Biostatistics, School of Public Health, Zahedan University of Medical Sciences, Zahedan,Iran
}

\section{A R T I C L E I N F O}

\section{Keywords:}

Seroprevalence

Hydatid cyst

Human

Zahedan

Iran

\begin{abstract}
A B S T R A C T
Introduction: Cystic echinococcosis (CE) or hydatidosis is one of the most common diseases between humans and livestock that humans are considered as an accidental host. This disease has spread globally, especially in the Mediterranean region, including Iran. To the best of our knowledge, no specific research has been conducted to investigate the epidemiological status of this disease in Zahedan, Sistan and Baluchestan Province, Iran. Therefore, this study was performed to achieve that aim for the first time.

Methods: A cross-sectional study was performed to evaluate the seroprevalence of hydatid cyst among patients (n = 773) referring to Zahedan health centers within 2019-2020. Zahedan health centers were classified into five geographical regions. The sampling process from each area was conducted using a simple randomized cluster method. The correlation between seropositivity and risk factors was evaluated using the Chi-square test.

Results: The seroprevalence of hydatid cyst in Zahedan city was estimated at 3.6\% (28 out of 773 samples). It was found that females were affected by this infection more than males, and the highest hydatid cyst seroprevalence rate was revealed in the age group of 31-50 years and the northeastern area of Zahedan. Although age, gender, vegetable consumption, soil contact had no significant influence on the risk of CE infection (P-value $>0.05)$, the relationship of keeping dogs in the household and the presence of stray dogs in a living environment with seropositivity to $\mathrm{CE}$ was significant (P-value $<0.05$ ).

Conclusion: The findings of this study showed that human hydatid cyst was common in Zahedan. Therefore, the implementation of preventive and hygienic principles, especially the presence of dogs in the household or stray dogs in the environment, should be a priority. Moreover, the various epidemiological aspects of this disease in Zahedan city should be taken into consideration.
\end{abstract}

\section{Introduction}

Cystic echinococcosis (CE), larvae infection of Echinococcus granulosus, is one of the most important zoonotic parasitic diseases common between livestock and human. ${ }^{1}$

Humans and livestock, respectively as an accidental and intermediate host, are infected by the digestion of infected vegetables and fruits. Dogs are the most important source of infection since they pass parasite eggs through the feces. ${ }^{2}$

The World Health Organization has recently identified it as a neglected disease. ${ }^{3}$

Cystic echinococcosis has a global spread, especially in Middle
Eastern countries, including Iran. ${ }^{1}$ Many studies have been conducted in different provinces of Iran, including Golestan, Arak, Chaharmahal and Bakhtiari, Kashan, Mazandaran, Isfahan, Khorasan Razavi, Fars, Hamedan and Azarbaijan, to determine the seroprevalence rate of human hydatid cyst. $^{4-13}$

The seroprevalence rate of human hydatid cyst in different provinces of Iran has been reported to range from $1.6 \%$ to $20 \% .^{11,14,15}$

The pooled seroprevalence rate of $\mathrm{CE}$ in Iran has been estimated at $6 \%{ }^{16}$ Zahedan is the center and capital of Sistan and Baluchestan province located in the southeast of Iran. This province is one of the deprived areas in Iran and has a hot, dry climate and poor hygiene. The seroprevalence rate and epidemiological status of human hydatid cyst in

\footnotetext{
* Corresponding author. Infectious Diseases and Tropical Medicine Research Center, Zahedan University of Medical Sciences, Zahedan, Iran.

E-mail address: abdolahi_s@zaums.ac.ir (S.A. Khabisi).
} 
this area are unknown. On the other hand, hydatid cyst risk factors have been presented in this area. Therefore, for the first time, this study was carried out to determine the seroprevalence rate of hydatid cyst among patients referring to Zahedan urban health centers.

\section{Methods}

\subsection{Area of study and sample collection}

This study, approved with the ethical code of IR.ZAUMS. REC.1398.137, was performed to evaluate the seroprevalence of hydatid cyst among 773 patients referring to Zahedan health centers $(n=44)$ within 2019-2020. Health centers were classified into five geographical areas, including northeast, northwest, southwest, southeast, and center with 12, 14, 8, 4, and 6 health centers, respectively. The sampling from each area was conducted using a simple randomized cluster method. The number of clusters from each geographical area was calculated based on the statistical formula. Blood samples were collected from 269 samples of 4 northeast clusters, 281 samples of 5 northwest clusters, 56 samples of 1 southeast cluster, 56 samples of 3 southwest clusters, and 111 samples of 2 center clusters. Subsequently, blood samples were collected from 773 participants after obtaining their consent to participate in the study and filling out the questionnaire. The patients who had a cyst removal surgery were excluded from the study. Blood samples were transferred to the parasitology laboratory of Zahedan University of Medical Sciences.

Sera were separated and stored at $-20{ }^{\circ} \mathrm{C}$ until further use.

\subsection{Detection of anti hydatid cyst antibodies using indirect ELISA}

The seroprevalence rate of the hydatid cyst was estimated with indirect ELISA using the recombinant B8/1 subunit of antigen B. This recombinant antigen was produced and evaluated (sensitivity 93\%, specificity $92 \%$ ) at Shiraz medical university. ${ }^{17}$ ELISA microplate was coated with $1.5 \mu \mathrm{g} / \mathrm{ml}$ of recombinant antigen B $(100 \mu \mathrm{l} /$ well) in $0.5 \mathrm{M}$ of carbonate-bicarbonate (pH: 9.5) coting buffer and incubated at $4{ }^{\circ} \mathrm{C}$, overnight. After five washing steps, $10 \%$ skimmed milk was used as a blocking solution. After five washing with PBST (phosphate buffer saline containing $0.05 \%$ tween 20 ), diluted sera $(1 / 100)$ in PBST were added and incubated for $1.5 \mathrm{~h}$ at room temperature. Also, positive and ten negative control serums (for calculation of cut off) confirmed with western blotting method were utilized. Five washing steps were carried out, and $100 \mu \mathrm{l} /$ well of horseradish peroxidase anti-human antibody $(1 / 2500$ dilution) was added and incubated for $1 \mathrm{~h}$ at room temperature. After five times washing, Tetra Methyl Benzidine (TMB) substrate (Cat. No: DB9510) was added (100 $\mu \mathrm{l} /$ well) and incubated out of light for 15 $\mathrm{min}$. Stop solution ( $0.2 \mathrm{M}$ of sulfuric acid) were added to each well, and absorbance at $450 \mathrm{~nm}$ and $650 \mathrm{~nm}$ (reference wave) were measured. Confirmed samples with the pathological method were used as positive controls serum. Cut off value was measured based on the mean OD of negative controls plus two standard deviations (SD).

\subsection{Statistical analysis}

The collected data were analyzed in SPSS software (version 22) using the Chi-square test to evaluate the relationship of participants' demographic data and risk factors with seropositivity to the hydatid cyst.

\section{Results}

Among the 773 participants, 520 (67.3\%) and 253 (32.7\%) were females and males, respectively. It was found that 28 out of the 773 subjects had antibodies against hydatid cyst recombinant antigen. Therefore, the seroprevalence rate of the human hydatid cyst was calculated at $3.6 \%$. More of the seropositive clients were housekeepers (2.5\%) and aged $31-50$ years. The most seropositive subjects (1.3\%) were detected in the northeast of Zahedan city. It was revealed that the factors of age, gender, soil contact, and vegetable consumption were not significantly effective in hydatid cyst infection (P-value $>0.05$ ). However, the relationship of keeping dogs in the household and the presence of stray dogs in the environment with seropositivity to hydatid was significant $(\mathrm{P}$-value $<0.05)$. Table 1 shows demographic information and relative hydatid cyst seropositivity among the patients referring to health centers of Zahedan city.

\section{Discussion}

Hydatid cyst is considered an important health problem and neglected disease. ${ }^{3}$ It has been spread worldwide, especially in Iran. ${ }^{1-}$ Various serological tests have been recognized as useful to identify asymptomatic and symptomatic infected individuals. However, most studies have used the ELISA method with antigen $B$ to this end. ${ }^{5-9}$ Although this antigen has high specificity and sensitivity, the application of hydatid cyst antigen $B$ related to a specific region is one of its most significant limitations. ${ }^{18,19}$

The recombinant antigen B subunit B8/1 with high sensitivity (92\%) and specificity (93\%) can be used to detect positive and suspicious cases. $^{17}$

In the present study, for the first time, the human seroprevalence rate of hydatid cyst in the urban areas of Zahedan city was reported as 3.6\%. The same seroprevalence rate has been reported in previous studies performed in other provinces, including Arak, Isfahan, East Azerbaijan, Karaj and Kerman. ${ }^{5,9,13,20,21}$ Inconsistent with the results of our study, a higher seroprevalence rate was reported from other areas of Iran including Mazandaran, Khuzestan, Lorestan and Yasuj. ${ }^{8,15,22,23}$ The reason for this discrepancy can be related to the fact that southeastern provinces of Iran, such as Sistan and Baluchestan, have a hot and dry climate; therefore, there are inadequate pastures and animal husbandry in such regions. In addition, the eggs of parasites are more sensitive to high temperatures and low humidity, which would explain the lower prevalence of hydatid cyst in this area.

The results of the present research indicated that females had a higher seroprevalence rate than males. Furthermore, housekeeper females were most infected, compared to other occupations. These results were in line with those of the other studies. ${ }^{11,24,25}$

Since women wash infected fruits and vegetables, they are more exposed to eggs of a parasite than men. However, in a recent study, no significant relationship was observed between gender and seropositivity to the hydatid cyst.

In the present study, the 31-50 year age group had the highest seroprevalence rate of hydatid cyst. The results of some previous studies were in agreement with this finding of the present research. ${ }^{4,5,26}$

The determination of age groups at the risk of hydatid cyst is challenging and different in studies owing to the long incubation period of this disease.

In a recent study, we reported a significant relationship of seropositivity to hydatid cyst with keeping dogs in the household and stray dog's presence in the environment. Dogs are considered the primary source of infection for humans and animals.

The results of the present research and the previous studies indicated the significant role of dogs in hydatid cyst prevalence. ${ }^{12}$

The highest level of seropositivity to the hydatid cyst was observed in the northeast of Zahedan city. The residents of this area suffer from poor hygienic and economic conditions. Additionally, the relationship between the geographical areas of Zahedan city and seropositivity to the hydatid cyst was not significant. We believe that the strengths of this study was the use of high efficiency recombinant antigen B subunit B8/ 1, in ELISA test. Due to the high sensitivity and specificity of this antigen, identifying suspicious patients with a low level of antibody titers against hydatid cyst was possible. Let's not forget that the serology tests may remain positive for a long period after resection of the cyst, therefore, to overcome this problem, patients with a history of liver cyst 
Table 1

Demographic data and relative hydatid cyst seropositivity in humans referred to health centers of Zahedan city, southeast Iran.

\begin{tabular}{|c|c|c|c|c|c|c|}
\hline \multirow[t]{2}{*}{ Characteristics } & & \multirow[t]{2}{*}{ Frequency } & \multirow[t]{2}{*}{ Percent } & \multicolumn{2}{|c|}{ seropositivity } & \multirow[t]{2}{*}{ Pvalue } \\
\hline & & & & Frequency & percent & \\
\hline \multirow[t]{2}{*}{ Sex } & Female & 520 & 67.3 & 22 & 4.2 & 0.13 \\
\hline & Male & 253 & 32.7 & 6 & 2.3 & \\
\hline \multirow[t]{4}{*}{ Age groups } & $<10$ & 29 & 3.7 & 1 & 3.5 & 0.4 \\
\hline & $10-30$ & 226 & 29.2 & 6 & 2.7 & \\
\hline & $31-50$ & 273 & 35.3 & 14 & 5.1 & \\
\hline & $>50$ & 245 & 31.8 & 7 & 2.9 & \\
\hline \multirow[t]{5}{*}{ Zahedan geographical area } & Central & 111 & 14.3 & 5 & 4.5 & 0.1 \\
\hline & Northeast & 269 & 35 & 10 & 3.7 & \\
\hline & Northwest & 281 & 36.3 & 8 & 3 & \\
\hline & Southeast & 56 & 7.2 & 0 & 0 & \\
\hline & Southwest & 56 & 7.2 & 5 & 9 & \\
\hline \multirow[t]{2}{*}{ Presence of dog in the household } & Yes & 268 & 34.7 & 18 & 6.8 & 0.001 \\
\hline & No & 505 & 65.3 & 10 & 2 & \\
\hline \multirow[t]{2}{*}{ Presence of stray dog in environment } & Yes & 378 & 49 & 9 & 2.4 & 0.05 \\
\hline & No & 395 & 51 & 19 & 8.4 & \\
\hline \multirow[t]{2}{*}{ Soil contact } & Yes & 691 & 89.4 & 27 & 3.9 & 0.18 \\
\hline & No & 82 & 10.6 & 1 & 1.2 & \\
\hline \multirow[t]{2}{*}{ Vegetable consumption } & Yes & 735 & 95 & 28 & 3.8 & 0.2 \\
\hline & No & 38 & 5 & 0 & 0 & \\
\hline \multirow[t]{6}{*}{ Job } & Rancher & 8 & 1 & 1 & 12.5 & 0.58 \\
\hline & Butcher & 2 & 0.2 & 0 & 0 & \\
\hline & Farmer & 1 & 0.1 & 0 & 0 & \\
\hline & Student & 109 & 14.2 & 3 & 2.7 & \\
\hline & housekeeper & 440 & 57 & 19 & 4.3 & \\
\hline & Other & 213 & 27.5 & 5 & 2.3 & \\
\hline
\end{tabular}

removal were excluded, this led to a decrease in false-positive results of the ELISA test. Moreover, for the first time, the seroprevalence of hydatid cyst was estimated in the southeast of Iran, which can be relatively helpful in clarifying the status of the CE in this region of Iran. Also, the following can be mentioned as weaknesses of the study. Imaging techniques play an important role in diagnosis and follow up of cystic echinococcosis but due to the covid19 pandemic situation, most of the seropositive individuals in this study, refused to come to clinic for imaging techniques. Ultrasound results of those who referred, indicated that there was a simple cyst in favor of hydatid cyst. Although, an expert committee of the WHO, working group on echinococcosis and Gharbi have suggested a standardized ultrasound classification of hepatic cystic echinococcosis which reveals cyst stages. ${ }^{27}$ This classification are not reported by the sonographer in Iran.

\section{Conclusion}

The findings of the study indicated that human hydatid cyst was a significant health problem in urban areas of Zahedan. It is recommended to observe the control and prevention principles of this disease and pay attention to its risk factors, including the presence and keeping of dogs. It also seems necessary to study the various epidemiological aspects of the disease in this region.

\section{Declaration of competing interest}

None.

\section{Acknowledgement}

The authors thank the participant for their cooperation and blood sample donation. This research was the subject of the MSc thesis of Malihe Marghzari. The study was financially supported by the office of vice-chancellor for research of Zahedan University of Medical Sciences (Grant No.9420).

\section{References}

1 Deplazes P, Rinaldi L, Alvarez Rojas CA, et al. Global distribution of alveolar and cystic echinococcosis. Adv Parasitol. 2017;95:315-493.

2 Mandal S, Mandal MD. Human cystic echinococcosis: epidemiologic, zoonotic, clinical, diagnostic and therapeutic aspects. Asian Pac J Trop Dis. 2012;5(4):253-260.

3 Brunetti E, Garcia HH, Junghanss T. Cystic echinococcosis: chronic, complex, and still neglected. PLoS Neglected Trop Dis. 2011;5(7):e1146.

4 Baharsefat M, Massoud J, Mobedi I, Farahnak A, Rokni M. Seroepidemiology of human hydatidosis in Golestan province, Iran. Iran J Parasitol. 2007;2(2):20-24.

5 Asghari M, Mohebali M, Eshrat Beigom K, et al. Seroepidemiology of human hydatidosis using AgB-ELISA test in Arak, Central Iran. Iran J Public Health. 2013;42 (4):391-396

6 Darani HY, Avijgan M, Karimi K, Manouchehri K, Masood J. Seroepidemiology of hydatid cyst in chaharmahal va Bakhtiari province, Iran. Iran J Public Health. 2003;32 (2):31-33.

7 Esmaeili N, Arbabi M. Seroepidemiology of hydatidosis among adult human at Kashan region, Iran in 2008. J Kashan Univ Med Sci. 2010;13(4):321-326.

8 Ziaei Hezarjaribi H, Fakhar M, Rahimi Esboei B, et al. Serological evidence of human cystic echinococcosis and associated risk factors among general population in Mazandaran Province, northern Iran. Ann Med Surg (Lond). 2017;18:1-5.

9 Ilbeigi P, Mohebali M, Kia EB, et al. Seroepidemiology of human hydatidosis using AgB-ELISA test in isfahan city and suburb areas, isfahan province, Central Iran. Iran J Public Health. 2015;44(9):1219-1224.

10 Khazaei S, Rezaeian S, Khazaei Z, et al. Epidemiological and clinical characteristics of patients with hydatid cysts in khorasan Razavi province, from 2011 to 2014 . Iran J Parasitol. 2016;11(3):364-370.

11 Sarkari B, Hosseini F, Abdolahi Khabisi S, Sedaghat F. Seroprevalence of cystic echinococcosis in blood donors in Fars province, southern Iran. Parasite Epidemiol Control. 2016;2(1):8-12.

12 Barati R, Sharifi-Sarasiabi K, Hamedi Y, Matini M, Shamseddin J. Seroprevalence of hydatidosis in kaboodarahang, hamadan province, Iran, in 2016 - 2017. Hormozgan Med J. 2018;22(4), e86498.

13 Garedaghi Y, Bahavarnia S. Seroepidemiology of human hydatidosis by ELISA method in East-Azarbaijan Province in Iran in year 2009. Iran J Epidemiol. 2011;7(2): 25-29.

14 Galeh TM, Spotin A, Mahami-Oskouei M, et al. The seroprevalence rate and population genetic structure of human cystic echinococcosis in the Middle East: a systematic review and meta-analysis. Int J Surg. 2018;51:39-48.

15 Rafiei A, Hemadi A, Maraghi S, Kaikhaei B, Craig PS. Human cystic echinococcosis in nomads of south-west Islamic Republic of Iran. East Mediterr Health J. 2007;13(1): $41-48$.

16 Shafiei R, Teshnizi SH, Kalantar K, Gholami M, Mirzaee G, Mirzaee F. The seroprevalence of human cystic echinococcosis in Iran: a systematic review and meta-analysis study. J Parasitol Res. 2016;2016, 1425147.

17 Savardashtaki A, Sarkari B, Arianfar F, Mostafavi-Pour Z. Immunodiagnostic value of Echinococcus granulosus recombinant B8/1 subunit of antigen B. Iran J Immunol. 2017;14(2):111-122.

18 Mohammadzadeh T, Sako Y, Sadjjadi SM, Sarkari B, Ito A. Comparison of the usefulness of hydatid cyst fluid, native antigen B and recombinant antigen B8/1 for 
serological diagnosis of cystic echinococcosis. Trans R Soc Trop Med Hyg. 2012;106 (6):371-375.

19 Rahimi H, Sadjjadi S, Sarkari B. Performance of antigen B isolated from different hosts and cyst locations in diagnosis of cystic echinococcosis. Iran J Parasitol. 2011;6 (1):12-19.

20 Dabaghzadeh H, Bairami A, Eshrat Beigom K, Aryaeipour M, Rokni MB. Seroprevalence of human cystic echinococcosis in Alborz Province, central Iran in 2015. Iran J Public Health. 2018;47(4):561-566.

21 Harandi M, Moazezi S, Saba M, et al. Sonographical and serological survey of human cystic echinococcosis and analysis of risk factors associated with seroconversion in rural communities of Kerman, Iran. Zoonoses Public Health. 2011;58(8):582-588.

22 Zibaei M, Azargoon A, Ataie-Khorasgani M, Ghanadi K, Sadjjadi S. The serological study of cystic echinococcosis and assessment of surgical cases during 5 years (20072011) in Khorram Abad, Iran. Niger J Clin Pract. 2013;16(2):221-225.
23 Sarkari B, Sadjjadi S, Beheshtian M, Aghaee M, Sedaghat F. Human cystic Echinococcosis in Yasuj district in Southwest of Iran: an epidemiological study of seroprevalence and surgical cases over a ten year period. Zoonoses public health. 2010;57(2):146-150.

24 Vlad DC, Neghina AM, Dumitrascu V, Marincu I, Neghina R, Calma CL. Cystic echinococcosis in children and adults: a seven-year comparative study in western Romania. Foodb Pathog Dis. 2013;10(2):189-195.

25 Possenti A, Manzano-Román R, Sánchez-Ovejero C, et al. Potential risk factors associated with human cystic echinococcosis: systematic review and meta-analysis. PLoS Neglected Trop Dis. 2016;10(11), e0005114.

26 Akalin S, Kutlu SS, Caylak SD, Onal O, Kaya S, Bozkurt Aİ. Seroprevalence of human cystic echinococcosis and risk factors in animal breeders in rural communities in Denizli, Turkey. J Infect Dev Ctries. 2014;8(9):1188-1194.

27 Brunetti E, Tamarozzi F, Macpherson C, et al. Ultrasound and cystic echinococcosis. Ultrasound Int Open. 2018;4(3):70-78. 